\title{
Multilevel Anterior Cervical Corpectomy and Fusion for Ossification of the Posterior Longitudinal Ligament
}

\author{
Zhichao Zhang \\ Shanghai Changzheng Hospital \\ Jingxuan Chen \\ Shanghai Changzheng Hospital \\ Wenmao Huang \\ Shanghai Changzheng Hospital \\ Yin Zhao \\ Shanghai Changzheng Hospital \\ Yanqing Sun \\ Shanghai Changzheng Hospital \\ Shengyuan Zhou \\ Shanghai Changzheng Hospital \\ Xiongsheng Chen ( $\nabla$ cxspine@smmu.edu.cn ) \\ Shanghai Changzheng Hospital \\ Lianshun Jia \\ Shanghai Changzheng Hospital
}

\section{Research article}

Keywords: Cervical OPLL, multilevel ACCF, Surgical outcomes, Complications

Posted Date: January 2nd, 2020

DOI: https://doi.org/10.21203/rs.2.19923/v1

License: @ (i) This work is licensed under a Creative Commons Attribution 4.0 International License. Read Full License 


\section{Abstract}

Background Cervical ossification of the posterior longitudinal ligament (OPLL) is a kind of spinal disorder widely spared in Asia. In dealing with multilevel cervical OPLL, the ideal surgical approach is still under debate. Here we introduce our findings of multilevel anterior cervical corpectomy and fusion (ACCF) in treating multilevel cervical OPLL

Methods 79 patients with multilevel cervical OPLL who had ACOR on C4-C5 or C5-C6 were retrospectively reviewed. Patients' Japanese Orthopedic Association (JOA) Score, the improvement rate of JOA Scores (RIS), sagittal cervical mobility (SCM) and the distance between the lower endplate of the vertebra body above the titanium mesh and the superior endplate of the vertebra body below the titanium mesh $(L$ ) were analyzed and subjected into paired-t test. Radiographic features and complications were described as well.

Results $p<0.001$ in all paired-t tests of JOA Scores, SCM and L at 1day, 6months, 1year and 2year follow-ups compared with preoperative ones. Patients' RIS continued to increase in 2 years. 6 (7.59\%) patients' L at the 2 years follow-up shortened more than $10 \mathrm{~mm}$ than their postoperative L. 1 (1.27\%) patient had severe titanium mesh canting.

Conclusions Multilevel ACCF procedure has positive outcomes in treating multilevel cervical OPLL. Postoperative complications like titanium subsidence and dislocation can happen after this procedure. Follow-ups should be conducted regularly and thoroughly with this kind of patients.

\section{Introduction}

Cervical ossification of the posterior longitudinal ligament (OPLL) is a kind of spinal disorder widely spared in Asia ${ }^{1}$. The etiology of the disease is still under debate ${ }^{2-3}$. The disease causes narrowing of the spinal canal and compression to the spinal cord which in turn leads to patients' neurological defections ${ }^{4}$. The ossification consumes the space reserve of the spinal canal through a very slow process. So patients with severe cervical OPLL can possess zero symptoms before fierce deterioration after mild triggers ${ }^{5}$. The disease can be easily misdiagnosed as cervical spondylotic myelopathy but should be dealt with more cautions ${ }^{6}$. Multilevel cervical OPLL, either continuous or segmental, may compress long range of spinal cord, cause spinal ischemia and syringomyelia and therefore lead to irreversible neurological damages ${ }^{7}$.

Surgical interventions for patients with cervical OPLL that aim to remove the ossification or decompress the spinal cord are of positive outcomes ${ }^{8}$. However, in dealing with multilevel cervical OPLL, surgeons have been arguing about the ideal surgical approach. Conventionally, a posterior approach of laminectomy surgery is popular among surgeons ${ }^{9}$. But the surgery will not remove the ossification mass which continues to grow thicker and longer. Also, a long segment posterior cervical fixation may affect patient's neck mobility and further lead to on-going neck pain ${ }^{10}$.

The anterior approach is another option which has been use more frequently in recent years. By conduct an ACCF while dissect the ossification mass, the surgeons may remove the lesion entirely and restore spinal stability ${ }^{11}$. However the anterior procedure usually demands more experience and skills for surgeons. The procedure may lead to residual of lesions, more intraoperative spinal cord harassments and increased risk of cerebrospinal fluid (CSF) leakage. Moreover, the problem of titanium mesh subsidence is one of the common complications as the origin bone defect after multilevel ACCF is remarkably large ${ }^{12}$. 
Our department has been conducting ACCF for years in treating cervical OPLL and acquired satisfying outcomes. We further classified it into two different procedures depending on the method of resecting the ossification lesions: conventional anterior cervical ossification piecemeal resection (ACOP) and anterior cervical ossification en-bloc resection (ACOE). ACCF on two continuous cervical segments on the other hand, presents more challenges for surgeons. So in light of such experiences, in the recent decade, we made attempt to perform a series of multilevel ACCF surgeries of C4-C5 or C5-C6 on patients with multilevel cervical OPLL. We collected and compared the related data of these patients and followed their status to explore the improvements in neurological symptoms, cervical mobility and titanium mesh subsidence after surgeries.

\section{Materials And Methods Patients}

From 2007 to 2017, a total 79 patients from our department had been included in this series. All of them had been diagnosed with multilevel cervical OPLL mainly occurred on C4-C6 based on their signs, symptoms and radiographic evidences. All patients had undergone through multilevel ACCF on C4-C5 or C5-C6 as the preoperative evaluations deemed them fitful.

Patients' medical records, preoperative and postoperative images were retrospectively reviewed and analyzed. Followups were conducted every 6 months for the first year after surgeries, then once a year thereafter. All patients' status was followed at least for 2 years. Cervical anterior, lateral, and dynamic X-rays were performed at each follow-up. Patients' information including general conditions, neurological function status and X-rays characteristics were reviewed and evaluated. The study was approved by the hospital's ethics committee. Informed consents were obtained from all the participants.

\section{Treatments and Surgical Techniques}

All patients received thorough assessments before surgeries, including physical examination, full series of blood tests, chest X-ray, abdominal ultrasound, electrocardiogram, and X-ray, computed tomography (CT) and magnetic resonance imaging (MRI) scan of the cervical spine.

Surgery indications were as follow: (1) Confirmed cervical OPLL with spinal cord compression along with intolerable pain/numbness or neurological defects. (2) Patient's symptoms were not significantly reduced or even deteriorated after conservative treatments. (3) Patient's general conditions were considered tolerable for surgical intervention. Patients with severe osteoporosis were excluded from this study. (4) Patients with ossification spinal canal occupying rate larger than $50 \%$ were scheduled to go through ACOE rather than ACOP to avoid spinal cord harassments. Individualized surgical strategy was designed mainly based on patients' signs and symptoms and radiographic evaluations.

For surgeries, the intervertebral discs above, between and below were removed along with the corpectomy resection of the vertebrae to expose the posterior cortices and the ossificated posterior longitudinal ligament. Pneumatic high speed burr was used to detach the edges of the ossification mass. The posterior interface with the dura sac was carefully separated by a cottle elevator. The entire ossification mass was removed in one piece as an en-bloc manner in ACOE, whereas the ossification was removed in smaller pieces in ACOP. Autogenous bones were chopped in pieces and filled into the titanium mesh. The titanium mesh, plate and fix screws were implanted at last (Fig. 1). The length of the titanium meshes we use were usually 5-8 $\mathrm{mm}$ longer than the patients' preoperative $L$ so as to acquire extra 
pressure on the bone graft interface. Additionally, if dura mater ossification was revealed in surgeries, the resection process was handled more delicately to prevent unnecessary CSF leakage.

Postoperatively, each patient was told to stay in bed for 3 days if not having intraoperative CSF leakage. Compression bandages around the neck were applied to patients with CSF leakage. Furthermore, a 3-months external immobilization with cervical-chest brace was advised to them when being discharged.

\section{Statistical analysis}

Attributes data are described by mean \pm standard deviation (range). Measurements data are described as counts (percentage). In this research, we defined the parameter $L$ as the distance between the lower endplate of the vertebra body above the titanium mesh and the superior endplate of the vertebra body below the titanium mesh. Factors including preoperative and postoperative Japanese Orthopedic Association (JOA) Score, the improvement rate of JOA Scores (RIS), sagittal cervical mobility (SCM) and L was calculated and analyzed. Patients were categorized into 4 groups according to RIS: excellent, greater than 75\%; good, 50-74\%; fair, 25-49\%; and poor, less than $25 \%$.

Paired t tests were used to compare the preoperative and postoperative data. A p value (two-sided) $\leq 0.05$ was considered statistically significant. All analyses were carried out using the SPSS for windows, version 22.0.0 (SPSS, IBM corp., New York, USA).

\section{Results}

\section{Preoperative Data}

79 multilevel cervical OPLL patients, including 57(72.15\%) male and 22(27.85\%) female, were included in this study. No patient died at our last follow-ups and no loss of follow-ups within the first 2 years. The average age was $57.0 \pm$ 8.96 (36-74). 7 (8.86\%) patients had acute trauma history, 32 (40.51\%) had neck and shoulder pain, $73(92.41 \%)$ had pain or numbness on the upper limbs and 46 (58.23\%) had lower limb weakness or sense of foot cotton. 46 (58.23\%) patients had positive Hoffmann's sign and 17 (21.52\%) had positive Babinski sign. The average preoperative JOA Scores were $10.97 \pm 2.10(4-14)$.

According to their preoperative radiographies, 38 (48.10\%) patients had segmental ossification and the other 41 (51.90\%) had continuous ossification. All patients suffered from combined intervertebral disc herniation, 30 (37.97\%) among them showed spinal hyperintensity on T2-weighted images of their MRI. The average Pavlov ratio of C4, 5 and 6 was $0.70 \pm 0.08(0.52-0.90)$, and the average occupying rate calculated on CT was $44.7 \pm 12.0 \%(23-71 \%)$. The average preoperative $L$ was $45.02 \pm 8.05 \mathrm{~mm}(27.9-59.7 \mathrm{~mm})$ and the average preoperative SCM was $28.31 \pm 11.67^{\circ}$ $\left(7.9-53.3^{\circ}\right)$. Figure 2 shows the preoperative and postoperative radiographic features of patient No.29 (patients listed in supplement materials).

\section{Surgical Outcomes and Complication Managements}

According to our records, 35 patients had ACOE and the other 44 had ACOP. 42 patients were operated on C5-C6 and the other 37 on C4-C5. All surgeries went smoothly. The average surgery time was $161.39 \pm 43.24 \mathrm{~min}$ (50-290 min). And the average blood loss was $170.78 \pm 100.82 \mathrm{ml}(50-2200 \mathrm{ml}) .2(2.53 \%)$ patients with intraoperative blood loss of $1000 \mathrm{ml}$ and $2200 \mathrm{ml}$ had blood transfusion.

Most patients state the alleviation of their preoperative symptoms after surgeries. 2 (2.53\%) patients showed signs of C5 palsy after surgeries but recovered over time. The average JOA Score on the first day postoperative was $11.64 \pm$ 
2.11 (5-15). The corresponding RIS was $11.03 \pm 15.19 \%$ (-25\%-50\%). RIS classification of excellent, good, fair and poor was $0 / 3 / 13 / 63$ respectively. Cervical X-rays were conducted to all patients in the meantime. The average postoperative $L$ was $50.31 \pm 10.14 \mathrm{~mm}(30.1-73.9 \mathrm{~mm})$.

$1(1.27 \%)$ patient had suffered from acute postoperative hematoma 10 hours after surgery, which then been dealt with an emergency second time surgery. 10 (12.66\%) patients had CSF leakage. Other than that, all patients had experienced smooth recoveries. Compression bandages around the neck were applied to patients with CSF leakage. All the leakages stopped within the first week. No other severe complications took place after surgeries. The average hospital stay time was $7.30 \pm 1.37$ day (5-14 day).

\section{Follow-ups}

We analyzed all patients' follow-ups at 6 months, 1 year and 2 years postoperative. Cervical X-rays were performed at each follow-up. Bony fusion was defined as the absence of radiolucent lines across the fusion site. All patients acquired successful bony fusion at the last follow up. Patients' JOA Scores postoperatively were significantly risen while their SCM obviously decreased. Although patients' L expanded after surgeries, we found a decreasing trend over time (Fig. 3 \& Table.1). RIS classification at 6 months, 1 year and 2 years follow-up were 0/7/22/50, 1/13/42/23 and 4/27/32/16 respectively (Fig. 4).

$2(2.53 \%)$ patients had another posterior laminectomy and fixation surgeries at 10 months and 4 years after the first surgeries respectively due to recurrence of the symptoms. No other patient complained about major recurrence or severe deterioration on their symptoms. However, 6 (7.59\%) patients' $L$ at the 2 years follow-up shortened more than $10 \mathrm{~mm}$ than their postoperative L. Additionally, 1 (1.27\%) patient (No.31) showed on his X-rays an obvious canting of the titanium mesh (Fig. 5).

\section{Discussion}

Surgical intervention is now considered the most ideal solution for advancing cervical OPLL. Ossification occurred on multiple continuous cervical segments however, requires more detailed and individualized preoperative evaluation to determine the best approach for the patient. A posterior laminectomy and fusion surgery in most cases is an acceptable alternative. But according to Chen's research, ACCF presents more optimal RIS than posterior laminectomy and fusion in treating multilevel cervical OPLL ${ }^{13}$.

Conventional ACOP requires less experience and skills in handling tools such as the pneumatic high speed burr which has the risk in injuring to the spinal cord. However ACOE clears the ossification more thorough and, for experienced surgeons, causes less harassment on the dura sac. Our department first reported similar procedure in treating thoracic ossification of the ligamenta flava ${ }^{14}$ and had been applied it to cervical OPLL in recent years. We held the opinion that ACOE is more suitable in patients with severe spinal cord compression (occupying rate $>50 \%$ ). Still, the choice of ACOE or ACOP to perform is flexible and needed to be decided individually.

From our series, the patients had significant increase in JOA Scores compared with preoperative, and continued to grow within our 2 year follow-ups. Also in the RIS classification analysis, the percentages of 'good' and 'excellent' grades had raised while the 'poor' grade decreased in 2 years after surgeries. These results indicate that multilevel ACCF possess positive outcomes in decompression the spinal cord and alleviating neurological symptoms in treating multilevel cervical OPLL. 
Postoperative hematoma happened in 1 patient in our series, the patient complained about difficulties in breathing and high tension around the neck region was observed. An emergency surgery was performed to release the pressure. Postoperative hematoma can lead to severe consequences if not noticed on time. Thorough hemostasis is required during this kind of surgeries to avoid such unwanted situation ${ }^{15}$. The incidence of CSF leakage in our series was $12.66 \%$, mostly happened on patients with dura matter ossification. It is trickier to perform ACCF on such kind of multilevel cervical OPLL. Sometimes damages on the dura sac are inevitable to acquire complete resection of the ossification. However, the method we used with compression bandages around the neck possessed ideal effect in stopping the leakage.

Satisfying fusion outcomes are harder to acquire with the surgically involved spinal segments increases ${ }^{16-17}$. Hence, it is an important phenomenon that bony fusion was observed in all patients of this series within the 2 year follow-up. Although patients' SCM significantly decreased after surgeries, which is an inevitable sacrifice, still there is approximately 20 degrees of total sagittal motion left in 2 years. This outcome proves that the implantation and the autogenous bone used in this procedure provide optimal spinal stability and avoid micro motions.

Titanium mesh subsidence is another major problem that needs to be avoided in this kind of surgery with long distance of bone loss ${ }^{18}$. To our experience, the subsidence process is inevitable before bony fusion is obtained. Titanium mesh subsidence may lead to fixation collapse or failure, kyphosis, continuous neck pain and even neurological defects. Patients with osteoporosis or not getting enough external fixation protection shortly after surgeries may end up with severe titanium mesh subsidence. The changes in the parameter $L$ in this research is indication the process of mesh subsidence. During our operations, we turned to use to titanium mesh a little longer $(5-8 \mathrm{~mm})$ than the patients' preoperative $L$ to make sure patients had an extra room for the mesh to sink before bony fusion arrived. Our results showed that patients' $L$ slowly shrank and almost equal to its preoperative value in 2 years.

Although methods in preventing mesh subsidence or dislocation had been conducted in operations, there are still patients presented with such complications in our follow-ups. 6 patients' titanium mesh sank more than $10 \mathrm{~mm}$ in 2 years and 1 patient had severe mesh canting. However despite the abnormalities on X-rays, these patients had no obvious neurological symptoms whatsoever. So we decided to put them under close observation rather than any aggressive remedies. Early time immobilization is crucial for patients who had this surgery especially the ones with osteoporosis. If patients have shown significant mesh dislocation and present with nonnegligible neurological symptoms, second time surgery is needed and posterior fixation is an acceptable choice.

\section{Conclusion}

We retrospectively reviewed a total of 79 patients with multilevel cervical OPLL who had multilevel ACCF procedure. The postoperative JOA Scores significantly increased over time and the RIS continued to increase in 2 years. In other word, multilevel ACCF procedure has positive outcomes for multilevel cervical OPLL patients. Patients' SCM will decrease after surgery as the consequence of bony fusion. The slight traction $(5-8 \mathrm{~mm})$ between the remaining vertebrae will benefit for preventing postoperative titanium mesh subsidence. Complications like titanium subsidence and dislocation can happen after this procedure. Close follow-ups shall be conducted regularly and thoroughly with this kind of patients.

\section{Abbreviations}

OPLL: ossification of the posterior longitudinal ligament; 
ACCF: anterior cervical corpectomy and fusion;

JOA: Japanese Orthopedic Association;

RIS: improvement rate of JOA Scores;

SCM: sagittal cervical mobility;

CSF: cerebrospinal fluid;

ACOP: anterior cervical ossification piecemeal resection;

ACOE: anterior cervical ossification en-bloc resection;

CT: computed tomography;

MRI: magnetic resonance imaging.

\section{Declaration}

\section{Ethics approval and consent to participate}

The research involved the use of hospital documentaries of 79 patients. This study was approved by our hospital's ethics committee and had been performed in accordance with the ethical standards as laid down in the 1964 Declaration of Helsinki and its later amendments. Informed consents were obtained from all the participants.

\section{Consent for publication}

Informed consent for publication was obtained from all individual participants for whom identifying information is included in this article (See in supplementary information files).

\section{Availability of data and material}

All data generated or analysed during this study are included in this published article (and its supplementary information files).

\section{Competing interests}

None.

\section{Funding}

None.

\section{Authors' contributions}

X.C., S.Z. and L.J. designed the study. Z.Z., J.C. and W.H. collected and analyzed the data. Z.Z., Y.S. and Y.Z. wrote the manuscript. All authors discussed the results and critically revised the manuscript.

\section{Acknowledgements}

None 


\section{References}

1. Liang, H.; Liu, G.; Lu, S.; Chen, S.; Jiang, D.; Shi, H.; Fei, Q., Epidemiology of ossification of the spinal ligaments and associated factors in the Chinese population: a cross-sectional study of 2000 consecutive individuals. BMC Musculoskelet Disord 2019, 20 (1), 253.

2. Abiola, R.; Rubery, P.; Mesfin, A., Ossification of the Posterior Longitudinal Ligament: Etiology, Diagnosis, and Outcomes of Nonoperative and Operative Management. Global Spine J 2016, 6 (2), 195-204.

3. Matsunaga, S.; Sakou, T., Ossification of the posterior longitudinal ligament of the cervical spine: etiology and natural history. Spine (Phila Pa 1976) 2012, 37 (5), E309-14.

4. Nishida, N.; Kanchiku, T.; Kato, Y.; Imajo, Y.; Suzuki, H.; Yoshida, Y.; Ohgi, J.; Chen, X.; Taguchi, T., Cervical ossification of the posterior longitudinal ligament: factors affecting the effect of posterior decompression. $J$ Spinal Cord Med 2017, 40 (1), 93-99.

5. Yan, L.; Gao, R.; Liu, Y.; He, B.; Lv, S.; Hao, D., The Pathogenesis of Ossification of the Posterior Longitudinal Ligament. Aging Dis 2017, 8 (5), 570-582.

6. Yoon, S. Y.; Park, T. H.; Eun, N. L.; Park, Y. G., The cutoff value of ossification of posterior longitudinal ligament (OPLL) for early diagnosis of myelopathy using somatosensory evoked potential in cervical OPLL patients. Spinal Cord 2017, 55 (6), 606-611.

7. Epstein, N., Diagnosis and surgical management of cervical ossification of the posterior longitudinal ligament. Spine J 2002, 2 (6), 436-49.

8. An, H. S.; Al-Shihabi, L.; Kurd, M., Surgical treatment for ossification of the posterior longitudinal ligament in the cervical spine. J Am Acad Orthop Surg 2014, 22 (7), 420-9.

9. Ohya, J.; Oshima, Y.; Oka, H.; Saiki, F.; Taniguchi, Y.; Matsubayashi, Y.; Tanaka, S.; Chikuda, H.; Takeshita, K., Patient Satisfaction with Posterior Decompression Surgery for Cervical Ossification of the Posterior Longitudinal Ligament: Prognostic Radiographic Factors and Patient-Reported Outcomes for the Effectiveness of Surgical Treatment. World Neurosurg 2016, 96, 272-279.

10. Takeuchi, K.; Yokoyama, T.; Numasawa, T.; Wada, K. I.; Itabashi, T.; Yamasaki, Y.; Kudo, H.; Ota, S., Fixed Neck Position in Multilevel Cervical Posterior Decompression and Fusion to Reduce Postoperative Disturbances of Cervical Spine Function. Spine Surg Relat Res 2018, 2 (4), 253-262.

11. Zhang, T.; Guo, Y.; Hu, N.; Chen, L.; Wu, Y.; Wang, Y.; Liu, L.; Zhao, C., Segmental Subtotal Corpectomy and Reconstruction With Titanium Cage and Anterior Plate for Multilevel Ossification of the Posterior Longitudinal Ligament. Orthopedics 2016, 39 (6), e1140-e1146.

12. Weber, M. H.; Fortin, M.; Shen, J.; Tay, B.; Hu, S. S.; Berven, S.; Burch, S.; Chou, D.; Ames, C.; Deviren, V., Graft Subsidence and Revision Rates Following Anterior Cervical Corpectomy: A Clinical Study Comparing Different Interbody Cages. Clin Spine Surg 2017, 30 (9), E1239-E1245.

13. Chen, Y.; Guo, Y.; Lu, X.; Chen, D.; Song, D.; Shi, J.; Yuan, W., Surgical strategy for multilevel severe ossification of posterior longitudinal ligament in the cervical spine. J Spinal Disord Tech 2011, 24 (1), 24-30.

14. Jia, L. S.; Chen, X. S.; Zhou, S. Y.; Shao, J.; Zhu, W., En bloc resection of lamina and ossified ligamentum flavum in the treatment of thoracic ossification of the ligamentum flavum. Neurosurgery 2010, 66 (6), 1181-6.

15. O'Neill, K. R.; Neuman, B.; Peters, C.; Riew, K. D., Risk factors for postoperative retropharyngeal hematoma after anterior cervical spine surgery. Spine (Phila Pa 1976) 2014, 39 (4), E246-52.

16. Vaccaro, A. R.; Falatyn, S. P.; Scuderi, G. J.; Eismont, F. J.; McGuire, R. A.; Singh, K.; Garfin, S. R., Early failure of long segment anterior cervical plate fixation. J Spinal Disord 1998, 11 (5), 410-5. 
17. Daubs, M. D., Early failures following cervical corpectomy reconstruction with titanium mesh cages and anterior plating. Spine (Phila Pa 1976) 2005, 30 (12), 1402-6.

18. van Jonbergen, H. P.; Spruit, M.; Anderson, P. G.; Pavlov, P. W., Anterior cervical interbody fusion with a titanium box cage: early radiological assessment of fusion and subsidence. Spine J 2005, 5 (6), 645-9; discussion 649.

\section{Table}

\begin{tabular}{cccccccccc}
\hline Preoperative & 1 Day & $\mathrm{p}$ & 6 Months & $\mathrm{p}$ & 1 Year & $\mathrm{p}$ & 2 Years & $\mathrm{p}$ \\
\hline $10.97 \pm 2.10$ & $11.64 \pm 2.11$ & $<0.001$ & $12.04 \pm 2.05$ & $<0.001$ & $12.78 \pm 1.91$ & $<0.001$ & $13.47 \pm 1.74$ & $<0.001$ \\
- & $11.03 \pm 15.19$ & - & $16.71 \pm 22.32$ & - & $30.15 \pm 19.43$ & - & $41.41 \pm 20.13$ & - \\
$44.98 \pm 8.05$ & $50.31 \pm 10.14$ & $<0.001$ & $48.62 \pm 9.26$ & $<0.001$ & $47.56 \pm 9.00$ & $<0.001$ & $46.94 \pm 8.99$ & $<0.001$ \\
$28.31 \pm 11.67$ & - & - & $17.45 \pm 8.27$ & $<0.001$ & $17.95 \pm 8.27$ & $<0.001$ & $18.29 \pm 9.25$ & $<0.001$ \\
\hline
\end{tabular}

Table.1. Patients' JOA Scores, RIS, $L$ and SCM at each time point. The $\mathrm{p}$ is the value in paired-t tests with preoperative group.

\section{Figures}

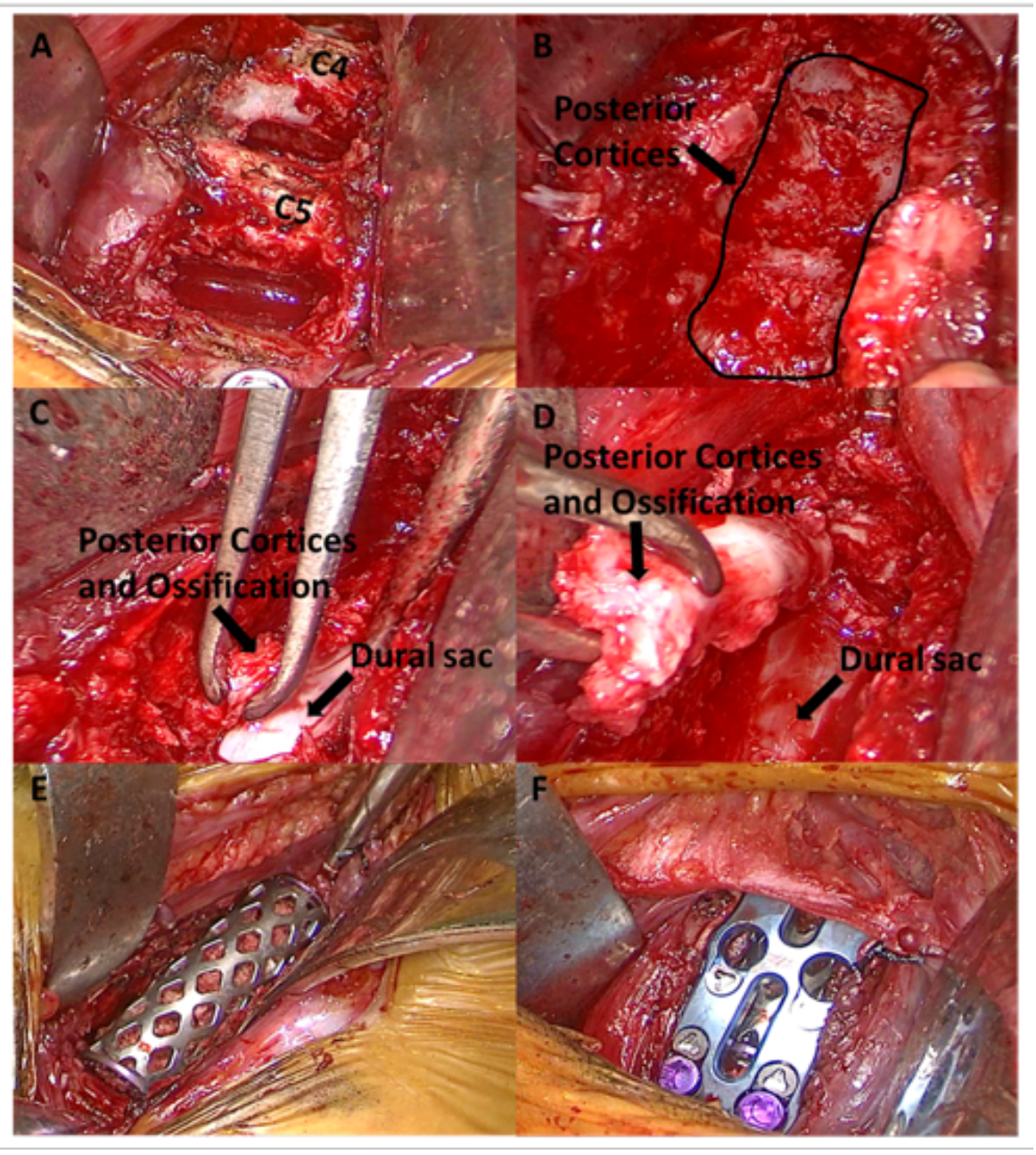

Figure 1 
Surgical procedures of multilevel ACOE. A. Remove intervertebral discs. B. Resect of the vertebrae, expose the posterior cortices and the ossification. C. Separate the anterior interface of the ossification. D. En-bloc removal of the ossification. E. Implanting titanium mesh. F. Implanting titanium plate and screws.
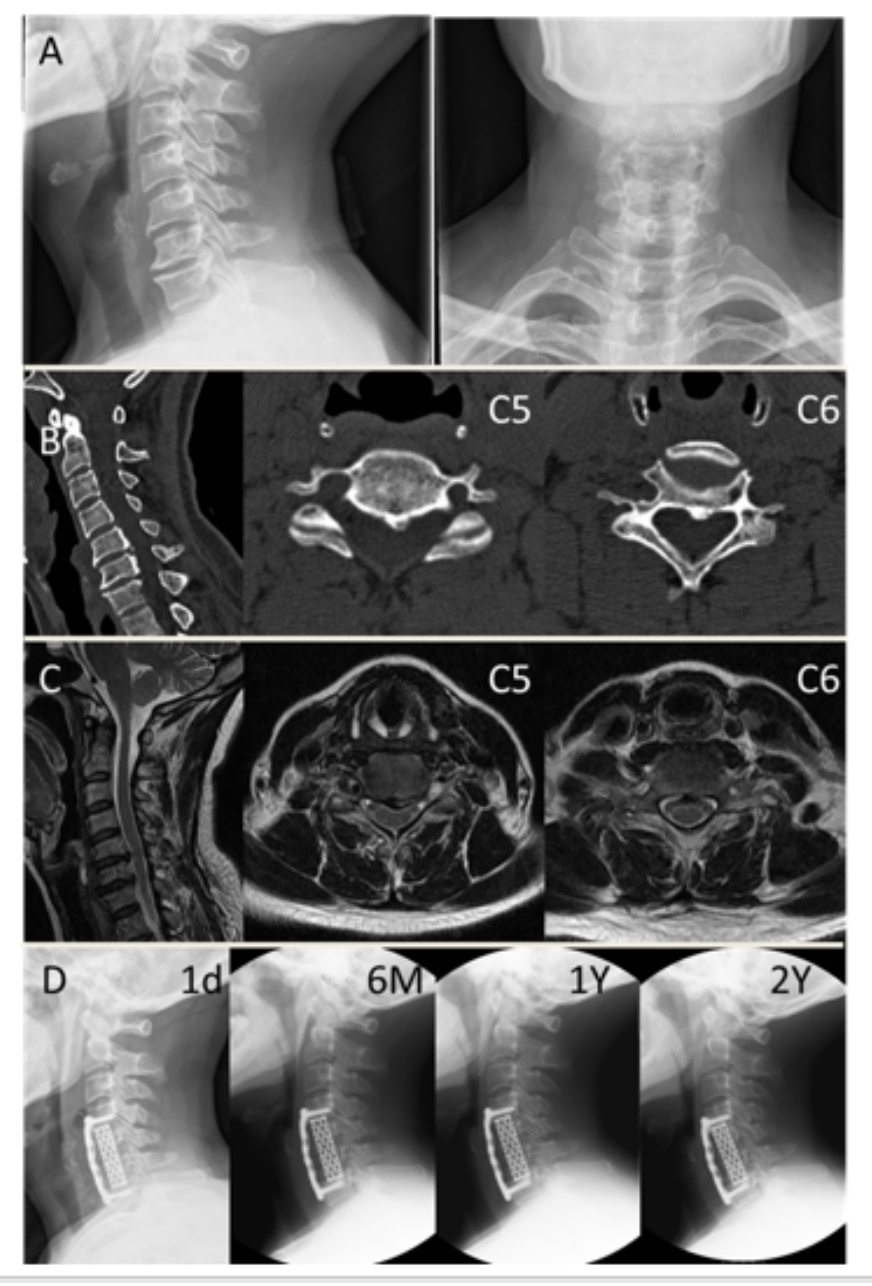

\section{Figure 2}

Radiographies of patient No.29. A. preoperative X-rays. B. preoperative CT. C. preoperative MRI. D. postoperative Xrays.
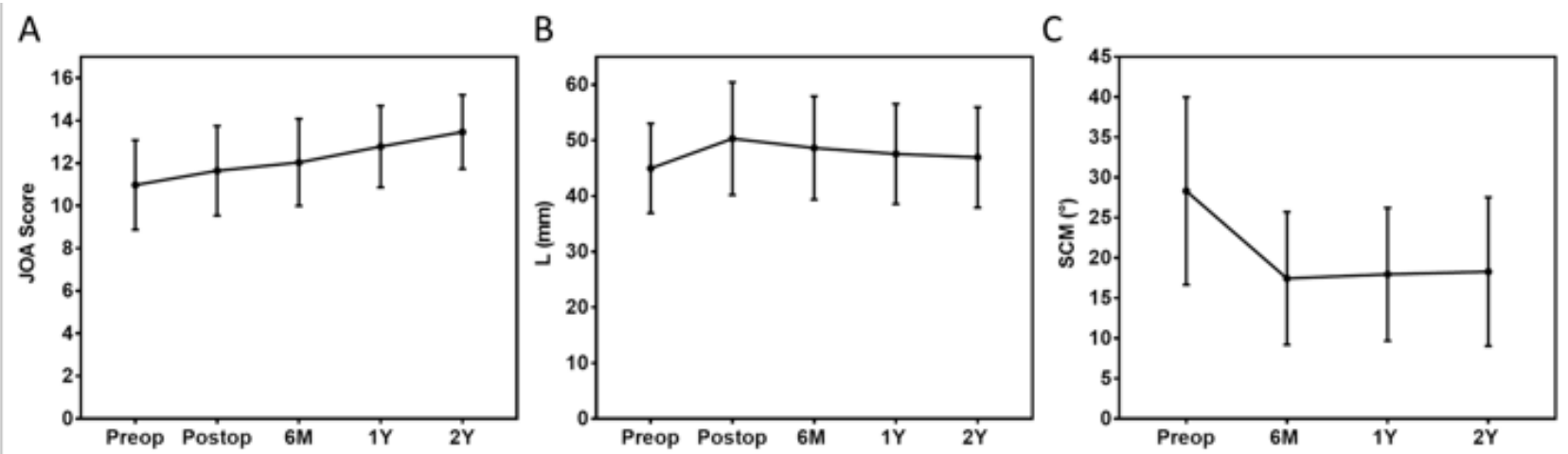

\section{Figure 3}

The changes of patients' JOA Scores, L and SCM at each time points. 

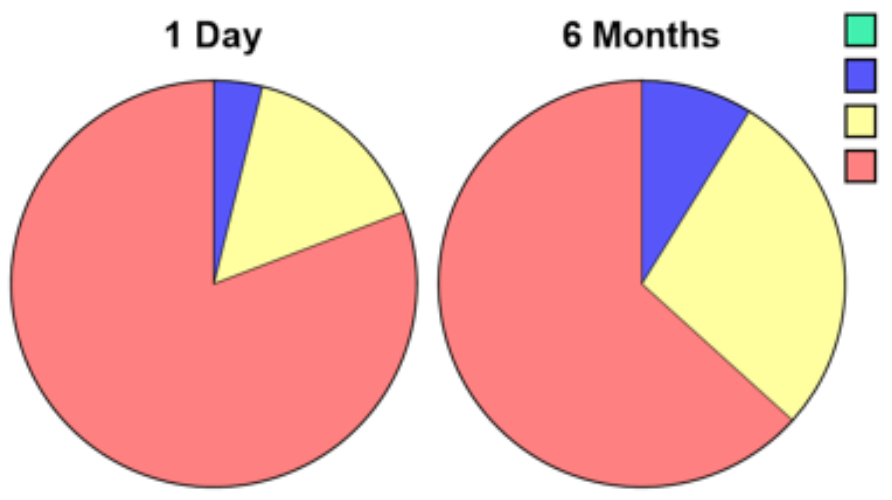

Excellent $(\geq 75 \%)$

Good (50-74\%)

Fair $(25-49 \%)$

Poor $(\leq 25 \%)$

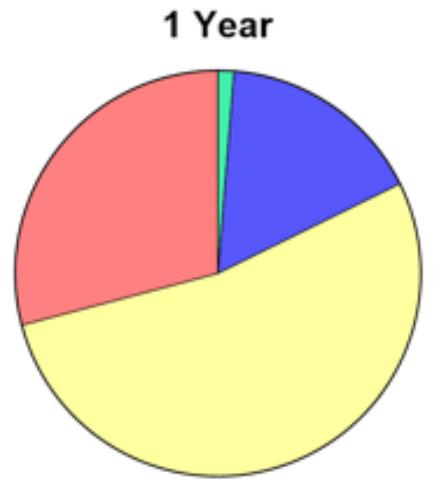

2 Years

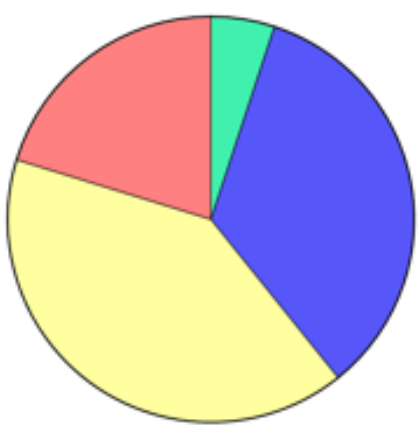

Figure 4

Pie charts of patients' RIS classification at each time points.

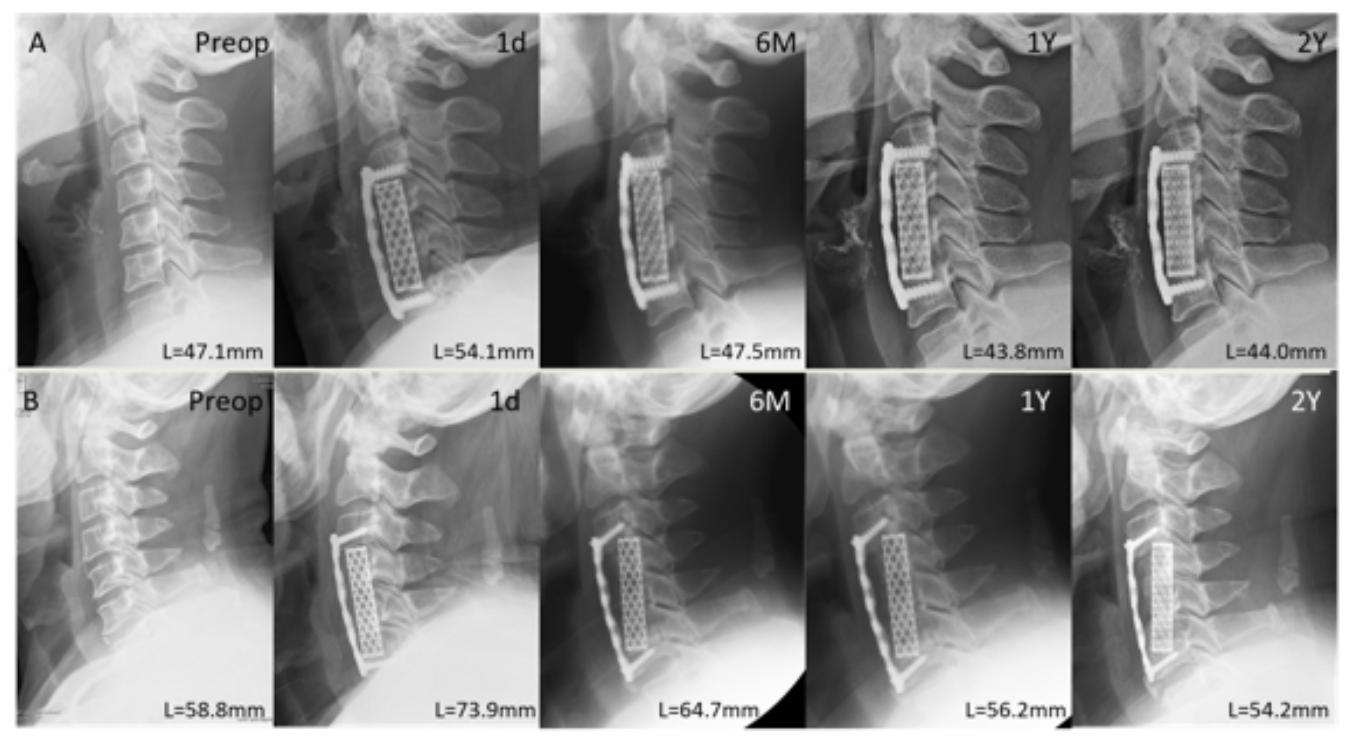

\section{Figure 5}

A. X-rays of patient No.41, L shortened more than $10 \mathrm{~mm}$ in 2 years. B. X-rays of patient No.31, significant titanium mesh canting.

\section{Supplementary Files}


This is a list of supplementary files associated with this preprint. Click to download.

- patientdetails.xlsx 